\title{
Improving 3GPP-LTE Uplink Control Signaling Performance Using Complex-Field Coding
}

\author{
Chaitanya Tumula and Erik G. Larsson
}

\section{Linköping University Post Print}

N.B.: When citing this work, cite the original article.

(C)2009 IEEE. Personal use of this material is permitted. However, permission to reprint/republish this material for advertising or promotional purposes or for creating new collective works for resale or redistribution to servers or lists, or to reuse any copyrighted component of this work in other works must be obtained from the IEEE.

Chaitanya Tumula and Erik G. Larsson, Improving 3GPP-LTE Uplink Control Signaling Performance Using Complex-Field Coding, 2012, IEEE Transactions on Vehicular Technology, (), , .

http://dx.doi.org/

Postprint available at: Linköping University Electronic Press http://urn.kb.se/resolve?urn=urn:nbn:se:liu:diva-80150 


\title{
Improving 3GPP-LTE Uplink Control Signaling Performance Using Complex-Field Coding
}

\author{
Tumula V. K. Chaitanya and Erik G. Larsson
}

\begin{abstract}
We study the uplink control signaling in 3GPPLong Term Evolution (LTE) systems. Specifically, we propose a precoding method that uses complex-field coding (CFC) to improve the performance of the physical uplink control channel (PUCCH) format 2 control signaling. We derive optimal detectors for both the conventional method and the proposed precoding method for different cases of channel state information (CSI) and noise variance information at the receiver. With a single receive antenna, the proposed method offers significant gains compared to the coding currently used in 3GPP-LTE for all the different scenarios considered in this work. However the gains are relatively less with two receive antennas.
\end{abstract}

Index Terms-3GPP-LTE, uplink control signaling, complexfield coding, perfect CSI, imperfect CSI, optimal detection.

\section{INTRODUCTION}

Fourth generation broadband wireless multiple access systems have data rate specifications in the order of hundreds of Mbit/sec (Mbps). For an LTE system with $20 \mathrm{MHz}$ bandwidth (BW), the targets for downlink (DL) and uplink (UL) peak data rate requirements are $100 \mathrm{Mbps}$ and $50 \mathrm{Mbps}$ respectively [1]. LTE uses orthogonal frequency division multiple access (OFDMA) for transmission in the downlink. In the uplink, in order to avoid large peak-to-average ratios, and to facilitate the use of more power-efficient RF amplifiers, LTE uses singlecarrier frequency division multiple access (SC-FDMA) [2].

The LTE system has separate channels both in the downlink and the uplink to carry control channel information (CCI). For example, the base station (eNodeB in LTE terminology) schedules different user equipments (UEs) in a single downlink frame. This scheduling information has to be sent to each of the UEs in a separate control channel to enable them to decode their data. In the uplink, the information about acknowledgment (ACK)/negative acknowledgment (NACK) for received downlink packets and also certain channel quality indicator (CQI) information have to be sent from each of the UEs to the eNodeB. The error performance of CCI is an important factor to improve the overall system performance, especially for cell-edge users who experience large path losses

Copyright (C) 2012 IEEE. Personal use of this material is permitted. However, permission to use this material for any other purposes must be obtained from the IEEE by sending a request to pubs-permissions@ieee.org.

The authors are with Linköping University, Dept. of Electrical Engineering (ISY), Division of Communication Systems, SE-581 83 Linköping, Sweden. (e-mail: \{tvk, erik.larsson\} @ isy.liu.se).

This work was supported in part by VINNOVA, the Swedish Foundation for Strategic Research (SSF), and ELLIIT. E. G. Larsson is a Royal Swedish Academy of Sciences (KVA) Research Fellow supported by a grant from the Knut and Alice Wallenberg Foundation.

Parts of the material in this paper were presented at the IEEE VTC 2010 conference [8].

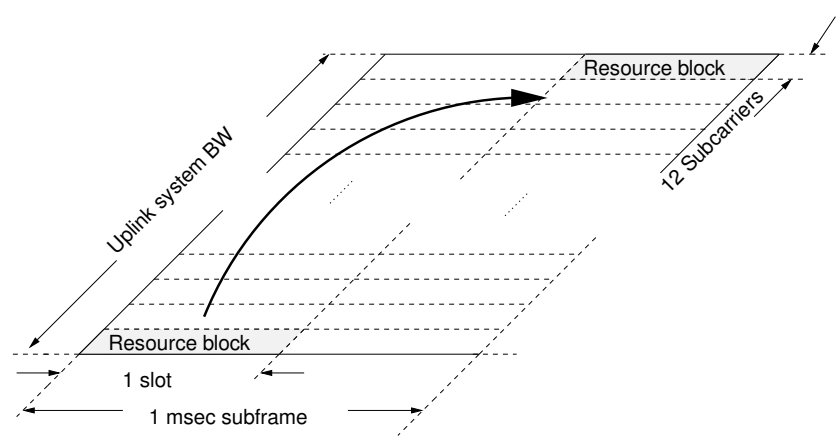

Figure 1. Uplink L1/L2 control signaling transmission on PUCCH (reproduced from [1, p. 398]).

and high inter-cell interference. In this paper, we focus on the uplink Layer 1/Layer 2 (L1/L2) control signaling in LTE. The uplink L1/L2 control signaling in LTE uses two different methods to send the uplink control data, depending on whether or not the UE has been assigned an uplink resource for uplink shared channel (UL-SCH) transmission, more details about control signaling transmission in LTE uplink can be found in [1]. In this work we focus on improving the performance of PUCCH format 2 control signaling. ${ }^{1}$

Fig. 1 shows the resources for uplink L1/L2 control signaling transmission on the PUCCH. These resources are located at the edges of the available bandwidth. Frequency hopping of these resources on the slot boundary provides frequency diversity to the control signaling. Each resource block consists of 12 OFDM subcarriers $\left(N_{\mathrm{sc}}^{\mathrm{RB}}\right)$ within each of two slots of an uplink subframe. The number of OFDM symbols in each slot of a subframe $\left(N_{\mathrm{symb}}^{\mathrm{RB}}\right)$ depends on the cyclic prefix (CP) length, see Table I.

\section{A. Related Work and Contributions}

The previous works on uplink control signaling in 3GPPLTE focused on different aspects of system performance. For PUCCH format 1 control signaling, multiuser receivers were proposed in [3], [4], and generalized likelihood ratio test (GLRT) based detectors were developed in [5]. A power boosting approach to improve the reliability of uplink control signaling was proposed in [6]. For PUCCH format 2 control signaling, robust multiuser channel estimators and multiuser detectors were presented in [7]. Most of the previous works on the uplink control signaling in LTE focused on the receiver

\footnotetext{
${ }^{1}$ Usually periodic CQI information reports are sent using this format. Sometimes simultaneous transmission of hybrid-automatic repeat request (ARQ) acknowledgments and CQI reports is also done using this format. This format can support a maximum of 13 information bits per subframe.
} 
design for specific scenarios. However, we show that one can achieve better error performance for PUCCH format 2 control signaling by applying precoding on the transmitter side.

In this work, we focus on the error protection for the CCI in the uplink of LTE. Specifically we are interested in the PUCCH format 2 control signaling which involves periodic reporting of CQI information separately or jointly with hybridautomatic repeat request (ARQ) acknowledgments. A (20, $N_{I}$ ) Reed-Muller code is used for control signaling using the PUCCH format 2 [10], where $N_{I}$ is the number of information bits and $N_{I} \leq 13 .^{2}$ Even though the control information is spread across two independent frequency bands (see Fig. 2), the specified code is not good at extracting the diversity mainly due to the short block length. To better extract this diversity and hence to improve the performance of the control signaling using the PUCCH format 2, we propose a method, where we precode pairs of modulated symbols selected from the two independent frequency bands. We use a $2 \times 2$ complex-field coding (CFC) matrix [12], [13] for precoding and then transmit the precoded data on the channel. ${ }^{3}$

In practical systems like LTE, the receiver will only have an estimate of the channel obtained from received pilots. The error in the channel estimate depends upon the channel estimation method used at the receiver. In this work, we derive and compare the performance of following detectors for both the conventional method and the proposed precoding method:

- Optimal detectors for perfect CSI and perfect noise variance knowledge at the receiver.

- Optimal non-coherent detectors, which only require statistical CSI and perfect noise variance knowledge at the receiver. ${ }^{4}$

- Optimal detectors for the case of channel estimation with perfect noise variance knowledge at the receiver. For this, we consider two practical channel estimation methods, namely

- minimum mean-square error (MMSE) channel estimation, and

- least-squares (LS) channel estimation.

- Optimal detectors for the case of channel estimation with unknown noise variance knowledge at the receiver.

Even though we focus on LTE uplink control signaling performance, the analysis presented in this work can be applied to any diversity combining system. The results show that, for the control signaling scenario in LTE as well, the traditional "mismatched detectors" obtained by plugging in the LS estimated channel in the coherent detection metric are inferior to the optimal detectors derived for the case with estimated channel gains [14], [15]. However, "mismatched detectors" which use an MMSE channel estimate have performance close

\footnotetext{
${ }^{2}$ In case of normal CP configuration, $N_{I}$ can only be $<11$. However in case of extended CP configuration, there is a provision to code both CQI report bits and hybrid-ARQ acknowledgment bits using the same Reed-Muller code. In this case $N_{I} \leq 13$.

${ }^{3}$ The proposed method may be applied in the scenarios (not only OFDM based systems), where the short codes over GF(2) cannot extract the diversity available in the channel resources.

${ }^{4}$ These detectors give an upper bound on the performance of receivers without perfect CSI.
}

Table I

RESOURCE BLOCK PARAMETERS FOR PUCCH FORMAT 2 TRANSMISSION

\begin{tabular}{|c|c|c|c|c|}
\hline Configuration & $N_{\mathrm{sc}}^{\mathrm{RB}}$ & $N_{\mathrm{symb}}^{\mathrm{RB}}$ & $\begin{array}{c}\text { Data symbol } \\
\text { indices }\end{array}$ & $\begin{array}{c}\text { Reference signal } \\
\text { symbol indices }\end{array}$ \\
\hline \hline Normal CP & 12 & 7 & $1,3,4,5,7$ & 2,6 \\
\hline Extended CP & 12 & 6 & $1,2,3,5,6$ & 4 \\
\hline
\end{tabular}

to that of the optimal non-coherent detector. The results also show that for all the different cases considered in this work, the proposed precoding method outperforms the conventional coding method suggested in LTE. However the gains for the proposed method reduce when the receiver has multiple antennas.

This work is an extension of our conference paper [8], in which we considered CFC based precoding with perfect CSI and using a simplified model for estimated channel. However, in this work, for the channel estimation case, we consider practical LS and MMSE channel estimation using the reference signals. In addition, we also study the performance of optimal non-coherent detectors as well as the performance with unknown noise variance.

\section{B. Organization of the Paper}

In Section II, we describe the system model for the PUCCH format 2 control signaling. We present the proposed precoding method in Section III. We derive the optimal detectors for perfect CSI with known noise variance case in Section IV, and optimal non-coherent detectors in Section V. Optimal detectors for the estimated channel case with known and unknown noise variance are presented in Sections VI, VII, respectively. Finally, we present simulation results in Section VIII and conclusions in Section IX.

\section{Notation}

Scalars are denoted with lower-case letters; Bold face lowercase and upper-case letters denote vectors and matrices respectively; $(.)^{T},(.)^{*},(.)^{H}$ denote transpose, complex conjugate, and the Hermitian operation respectively; $\mathbb{E}[$.$] denotes$ the expectation operator; $\mathcal{C N}(\mathbf{x}, \mathbf{C})$ represents a circularly symmetric complex Gaussian vector distribution with mean $\mathbf{x}$ and covariance matrix $\mathbf{C}$; $\operatorname{diag}($.$) denotes a diagonal matrix$ and $\|$.$\| denotes the Euclidean norm.$

\section{SySTEM ModeL}

CQI reports from the UE to eNodeB are useful for channeldependent scheduling in the downlink. A CQI report consists of a maximum of 11 information bits per subframe [10]. Since PUCCH format 1 can support at most two information bits per subframe, CQI information reports on $\mathrm{PUCCH}$ are sent using the PUCCH format 2. The structure of $P U C C H$ format 2 depends on the $\mathrm{CP}$ configuration. Table I summarizes the configuration-dependent resource block parameters. Fig. 2 illustrates the PUCCH format 2 for the case of normal CP. The CQI information bits are coded using the $\left(20, N_{I}\right)$ ReedMuller code generator matrix specified in [10], and the 20 


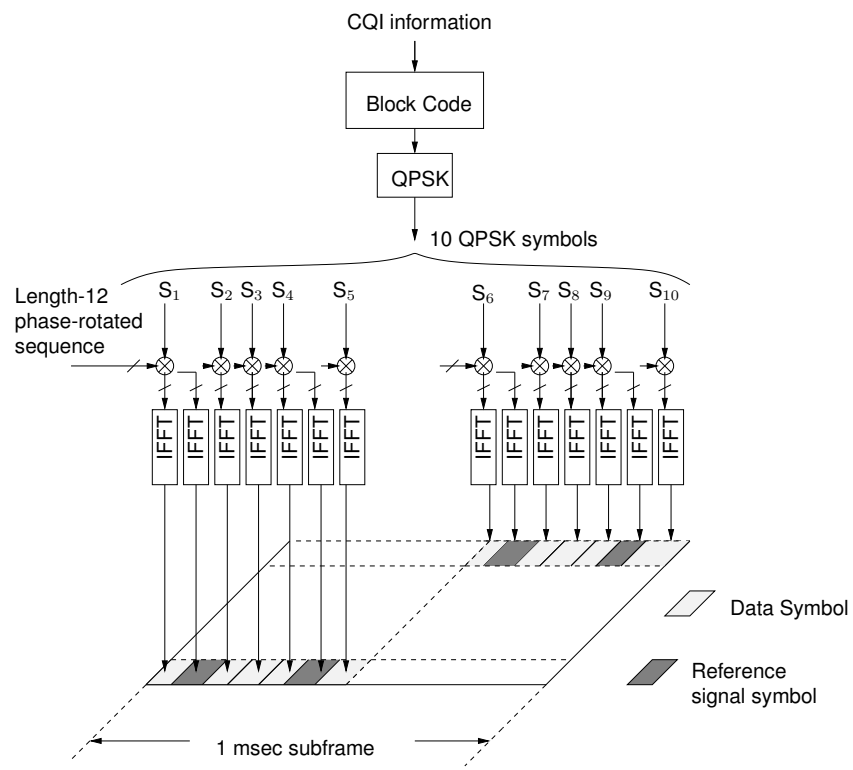

Figure 2. PUCCH format 2 for normal CP (reproduced from [1, p. 406]).

coded output bits are modulated using a quadrature phase shift keying (QPSK) constellation $(\mathcal{S}){ }^{5}$

Let $\mathbf{b}_{I}=\left[b_{1}, b_{2}, \cdots, b_{N_{I}}\right]^{T}$ be the vector of CCI information bits and let $\mathbf{G}$ denote the generator matrix of the Reed-Muller code. We write the coded output bit vector $\mathbf{b}_{O}=\left[b_{1}, b_{2}, \cdots, b_{20}\right]^{T}$ as $\mathbf{b}_{O}=\mathbf{G b}_{I}$. Let $s_{1}, s_{2}, \cdots, s_{10}$ be the resulting QPSK symbols. The first five QPSK symbols $s_{1}, s_{2}, \cdots, s_{5}$ are transmitted in the first slot and the remaining five symbols $s_{6}, s_{7}, \cdots, s_{10}$ are transmitted in the last slot of a subframe. There are seven OFDM symbols in each slot. Two of them are used for reference signals to facilitate coherent demodulation. Each of the five QPSK data symbols is spread across the subcarriers in each symbol of the resource block by using a length-12 phase-rotated cell-specific sequence. Details about the phase-rotation sequence can be found in [11].

We assume that both the transmitter and receiver are equipped with single antenna. We also assume that the channel gains are constant in one time slot, but change from one time slot to the next one. To simplify notation, we assume that the OFDM symbols which carry the QPSK symbols are contiguous (there are no reference signal symbols between them). Let $N_{\text {symb }}^{\text {data }}$ denote the number of data symbols in one subframe. For PUCCH format 2, $N_{\mathrm{symb}}^{\mathrm{data}}=10$ (independently of the $\mathrm{CP}$ configuration). At the receiver, after the fast Fourier transform (FFT) operation and after undoing the effect of the phase rotation sequence, the received signal in slot $l \in\{1,2\}$ over the $N_{\mathrm{sc}}^{\mathrm{RB}}$ subcarriers can be written as:

$$
\mathbf{Y}_{l}=\left[\begin{array}{ll}
\mathbf{Y}_{l}^{p} & \mathbf{Y}_{l}^{s}
\end{array}\right]=\mathbf{h}_{l}\left[\begin{array}{ll}
\mathbf{d}_{p}^{T} & \mathbf{d}_{l}^{T}
\end{array}\right]+\underbrace{\left[\begin{array}{ll}
\mathbf{W}_{l}^{p} & \mathbf{W}_{l}^{s}
\end{array}\right]}_{\triangleq \mathbf{W}_{l}},
$$

where

- $\mathbf{Y}_{l}$ is the $N_{\mathrm{sc}}^{\mathrm{RB}} \times N_{\mathrm{symb}}^{\mathrm{RB}}$ matrix of received signal in slot $l$.

${ }^{5}$ The LTE standard specifies a UE specific scrambling sequence to scramble the coded bits before modulating the data [11]. However since the performance is independent of the scrambling sequence, we do not consider any scrambling sequence in this paper.
- $\mathbf{Y}_{l}^{p} \triangleq\left[\mathbf{y}_{l 1}^{p}, \mathbf{y}_{l 2}^{p}\right]$ is the $N_{\mathrm{sc}}^{\mathrm{RB}} \times 2$ matrix of received signals on reference symbols in slot $l$.

- $\mathbf{Y}_{1}^{s} \triangleq\left[\mathbf{y}_{1}, \mathbf{y}_{2}, \ldots, \mathbf{y}_{5}\right]$ is the $N_{\mathrm{sc}}^{\mathrm{RB}} \times N_{\mathrm{symb}}^{\text {data }} / 2$ matrix of received signals on all $N_{\mathrm{sc}}^{\mathrm{RB}}$ subcarriers of data symbols 1 to 5 in slot 1 .

- $\mathbf{Y}_{2}^{s} \triangleq\left[\mathbf{y}_{6}, \mathbf{y}_{7}, \ldots, \mathbf{y}_{10}\right]$ is the $N_{\mathrm{sc}}^{\mathrm{RB}} \times N_{\mathrm{symb}}^{\text {data }} / 2$ matrix of received signals on all $N_{\mathrm{sc}}^{\mathrm{RB}}$ subcarriers of data symbols 6 to 10 in slot 2 .

- $\mathbf{h}_{l} \triangleq\left[h_{1 l}, h_{2 l}, \ldots, h_{N_{\mathrm{sc}}^{\mathrm{RB}} l}\right]$ is the frequency-domain channel vector in slot $l .^{6}$

- $\mathbf{d}_{1}^{T}=\left[s_{1}, s_{2}, \ldots, s_{5}\right]^{T}$ denote the first five QPSK symbols modulated in slot 1 .

- $\mathbf{d}_{2}^{T}=\left[s_{6}, s_{7}, \ldots, s_{10}\right]^{T}$ denote the last five QPSK symbols modulated in slot 2 .

- $\mathbf{W}_{l}^{p} \triangleq\left[\mathbf{w}_{l 1}^{p}, \mathbf{w}_{l 2}^{p}\right]$ is the $N_{\mathrm{sc}}^{\mathrm{RB}} \times 2$ matrix of additive noise samples on reference symbols in slot $l$.

- $\mathbf{W}_{1}^{s} \triangleq\left[\mathbf{e}_{1}, \mathbf{e}_{2}, \ldots, \mathbf{e}_{5}\right]$ is the $N_{\mathrm{sc}}^{\mathrm{RB}} \times N_{\mathrm{symb}}^{\text {data }} / 2$ matrix of additive noise samples on all $N_{\mathrm{sc}}^{\mathrm{RB}}$ subcarriers of data symbols 1 to 5 in slot 1 .

- $\mathbf{W}_{2}^{s} \triangleq\left[\mathbf{e}_{6}, \mathbf{e}_{7}, \ldots, \mathbf{e}_{10}\right]$ is the $N_{\mathrm{sc}}^{\mathrm{RB}} \times N_{\text {symb }}^{\text {data }} / 2$ matrix of additive noise samples on all $N_{\mathrm{sc}}^{\mathrm{RB}}$ subcarriers of data symbols 6 to 10 in slot 2 .

We assume $\mathbf{d}_{p}^{T} \triangleq\left[s_{p_{1}}, s_{p_{2}}\right]^{T}$ is the vector of transmitted pilot symbols in both the slots. ${ }^{7}$ We also assume that all the entries of $\mathbf{W}_{l}$ are i.i.d. with distribution $\mathcal{C N}\left(0, N_{0}\right)$. Note that, we can write the received signal on data symbols as: ${ }^{8}$

$$
\mathbf{y}_{m}=\mathbf{h}_{\left\lceil\frac{m}{5}\right\rceil} s_{m}+\mathbf{w}_{m}, \quad 1 \leq m \leq N_{\text {symb }}^{\text {data }}
$$

\section{Proposed Method for Control Signaling USing PRECODING}

The Reed-Muller code with short block length used for control signaling is not able to extract all of the available frequency diversity. To extract more of this frequency diversity inherent in the resources for PUCCH format 2, we apply precoding on pairs of symbols from two independent slots of a subframe. More specifically, we transmit $x_{m}$ instead of $s_{m}$, where $x_{m}$ are obtained by a linear transformation of pairs of $s_{m}$ as follows:

$$
\underbrace{\left[\begin{array}{c}
x_{m} \\
x_{m+5}
\end{array}\right]}_{\triangleq \mathbf{x}}=\underbrace{\left[\begin{array}{ll}
\psi_{11} & \psi_{12} \\
\psi_{21} & \psi_{22}
\end{array}\right]}_{\triangleq \Psi} \underbrace{\left[\begin{array}{c}
s_{m} \\
s_{m+5}
\end{array}\right]}_{\triangleq \mathbf{s}}, 1 \leq m \leq \frac{N_{\mathrm{symb}}^{\text {data }}}{2}
$$

For precoding, we use a $2 \times 2$ CFC matrix $\Psi$ generated using the designs specified in [12], [13]. It was shown that these designs provide full diversity (diversity of 2 in the present case). The key point is that the precoder improves the minimum product distance, which determines the performance

\footnotetext{
${ }^{6}$ Note that the analysis presented in this paper is independent of the fading distribution of the channel. However, for the numerical results, we assume a tapped-delay-line channel model with Rayleigh fading taps.

${ }^{7}$ Note that for the extended $\mathrm{CP}$ configuration, there is only one reference signal symbol in each time slot as shown in Table I. For extended CP case, $d_{p}^{T}=s_{p}$ is a scalar. Throughout the paper, we present the analysis for the normal CP case with two reference signal symbols.

${ }^{8}$ Note that $\left\lceil\frac{m}{5}\right\rceil$ can only take values 1 and 2 as $1 \leq m \leq N_{\text {symb }}^{\text {data }}=10$. All the five data symbols in first time slot experience the channel gain vector $\mathbf{h}_{1}$ and the remaining five data symbols in the second time slot experience $\mathbf{h}_{2}$.
} 
in fading channels. We consider only unitary precoders, so that the performance on the AWGN channel is unaffected. ${ }^{9}$ The transmit power also remains constant with the unitary precoder, because $\|\mathbf{s}\|^{2}=\|\mathbf{\Psi} \mathbf{s}\|^{2}$. With the precoding, the combined received signal vector after undoing the effect of phase rotation sequence on all the subcarriers of OFDM symbols $m$ and $(m+5)$ can be written as:

$$
\begin{aligned}
\underbrace{\left[\begin{array}{c}
\mathbf{y}_{m} \\
\mathbf{y}_{m+5}
\end{array}\right]}_{\triangleq \mathbf{y}} & =\underbrace{\left[\begin{array}{cc}
\mathbf{h}_{1} & \mathbf{0} \\
\mathbf{0} & \mathbf{h}_{2}
\end{array}\right]}_{\triangleq \mathbf{F} \triangleq\left[\mathbf{f}_{1}, \mathbf{f}_{2}\right]}\left[\begin{array}{ll}
\psi_{11} & \psi_{12} \\
\psi_{21} & \psi_{22}
\end{array}\right]\left[\begin{array}{c}
s_{m} \\
s_{m+5}
\end{array}\right]+ \\
& \underbrace{\left[\begin{array}{c}
\mathbf{w}_{m} \\
\mathbf{w}_{m+5}
\end{array}\right]}_{\triangleq \mathbf{w}}, \quad 1 \leq m \leq \frac{N_{\mathrm{symb}}^{\text {data }}}{2} \\
= & \mathbf{F} \mathbf{\Psi} \mathbf{s}+\mathbf{w}=\mathbf{F} \mathbf{x}+\mathbf{w} .
\end{aligned}
$$

Throughout this work, we use the following unitary $\Psi$ matrix [13]

$$
\Psi=\left[\begin{array}{cc}
\frac{1}{\sqrt{2}} & \frac{1}{2}-j \frac{1}{2} \\
\frac{1}{\sqrt{2}} & -\frac{1}{2}+j \frac{1}{2}
\end{array}\right]
$$

\section{Optimal Detectors for Perfect CSI AND KnOWN $N_{0}$}

In this section, we derive the optimal detectors for the conventional method and the proposed precoding method under the assumption of perfect CSI and perfect knowledge of noise variance value $N_{0}$ at the receiver.

\section{A. Conventional Coding Case}

For the conventional coding case, the optimal detector for $s_{m}, 1 \leq m \leq N_{\text {symb }}^{\text {data }}$, is obtained by maximizing the conditional probability $p\left(\mathbf{y}_{m} \mid \mathbf{h}_{\left\lceil\frac{m}{5}\right\rceil}, s_{m}, N_{0}\right)$. It can be shown that:

$$
p\left(\mathbf{y}_{m} \mid \mathbf{h}_{\left\lceil\frac{m}{5}\right\rceil}, s_{m}, N_{0}\right)=\frac{1}{\left(\pi N_{0}\right)^{N_{\mathrm{sc}}^{\mathrm{RB}}}} \exp \left(-\frac{\left\|\mathbf{y}_{m}-\mathbf{h}_{\left\lceil\frac{m}{5}\right\rceil} s_{m}\right\|^{2}}{N_{0}}\right) .
$$

Maximizing the conditional probability in (6) is equivalent to:

$$
\min _{s_{m} \in \mathcal{S}}\left\|\mathbf{y}_{m}-\mathbf{h}_{\left\lceil\frac{m}{5}\right\rceil} s_{m}\right\|^{2} \Longleftrightarrow \min _{s_{m} \in \mathcal{S}}\left|s_{m}-\hat{s}_{m}\right|^{2},
$$

where

$$
\hat{s}_{m} \triangleq \frac{\mathbf{h}_{\left\lceil\frac{m}{5}\right\rceil}^{H} \mathbf{y}_{m}}{\left\|\mathbf{h}_{\left\lceil\frac{m}{5}\right\rceil}\right\|^{2}}=s_{m}+\frac{\mathbf{h}_{\left\lceil\frac{m}{5}\right\rceil}^{H} \mathbf{w}_{m}}{\left\|\mathbf{h}_{\left\lceil\frac{m}{5}\right\rceil}^{H}\right\|^{2}}=s_{m}+\tilde{w}_{m}
$$

and where $\tilde{w}_{m} \sim \mathcal{C N}\left(0, \frac{N_{0}}{\left\|\mathbf{h}_{\left[\frac{m}{5}\right\rceil}^{H}\right\|^{2}}\right)$. Equation (8) corresponds to maximal-ratio-combining (MRC) at the receiver. Using the fact that $s_{m} \in \mathcal{S}$, assuming that all bits are a priori independent, and assuming equal a priori probabilities

$$
{ }^{9} \text { Note that if } \Psi^{H} \mathbf{\Psi}=\mathbf{I} \text {, then }\left\|\mathbf{\Psi}\left(\mathbf{s}-\mathbf{s}^{\prime}\right)\right\|=\left\|\mathbf{s}-\mathbf{s}^{\prime}\right\| \forall \mathbf{s}, \mathbf{s}^{\prime} \in \mathcal{S}^{2} .
$$

for the bits that constitute $s_{m}$, the a posteriori log-likelihood ratio (LLR) for the information bits that constitute $s_{m}$ can be written as:

$$
L\left(b_{i} \mid \mathbf{y}_{m}\right)=\log \left(\frac{\sum_{s_{m}: b_{i}\left(s_{m}\right)=1} \exp \left(-\frac{\left\|\mathbf{h}_{\left\lceil\frac{m}{5}\right\rceil}\right\|^{2}}{N_{0}}\left|s_{m}-\hat{s}_{m}\right|^{2}\right)}{\sum_{s_{m}: b_{i}\left(s_{m}\right)=0} \exp \left(-\frac{\left\|\mathbf{h}_{\left\lceil\frac{m}{5}\right\rceil}\right\|_{0}^{2}}{N_{0}}\left|s_{m}-\hat{s}_{m}\right|^{2}\right)}\right) .
$$

\section{B. Proposed Precoding Case}

In the proposed precoding case, the optimal detector for $\mathbf{s}$ is obtained by maximizing the conditional distribution of $\mathbf{y} \mid \mathbf{F}, \boldsymbol{\Psi}, \mathbf{s}, N_{0}$, which is given by:

$$
p\left(\mathbf{y} \mid \mathbf{F}, \mathbf{\Psi}, \mathbf{s}, N_{0}\right)=\frac{1}{\left(\pi N_{0}\right)^{2 N_{\mathrm{sc}}^{\mathrm{RB}}}} \exp \left(-\frac{\|\mathbf{y}-\mathbf{F} \mathbf{\Psi} \mathbf{s}\|^{2}}{N_{0}}\right) .
$$

Maximizing (10) is the same as:

$$
\min _{\mathbf{s} \in \mathcal{S}^{2}}\|\mathbf{y}-\mathbf{F} \mathbf{\Psi} \mathbf{s}\|^{2} .
$$

Let the $\mathrm{QR}$ decomposition of $\mathbf{F}$ be given by $\mathbf{F}=\mathbf{Q R}$, where $\mathbf{Q}$ is a $2 N_{\mathrm{sc}}^{\mathrm{RB}} \times 2$ semi-unitary matrix $\left(\mathbf{Q}^{H} \mathbf{Q}=\mathbf{I}\right)$ and $\mathbf{R}$ is a $2 \times 2$ upper triangular matrix. Owing to the structure of $\mathbf{F}$, it turns out that

$$
\mathbf{Q}=\left[\frac{\mathbf{f}_{1}}{\left\|\mathbf{f}_{\mathbf{1}}\right\|}, \frac{\mathbf{f}_{2}}{\left\|\mathbf{f}_{\mathbf{2}}\right\|}\right]
$$

and

$$
\mathbf{R}=\operatorname{diag}\left(\left\|\mathbf{f}_{1}\right\|,\left\|\mathbf{f}_{2}\right\|\right)=\operatorname{diag}\left(\left\|\mathbf{h}_{1}\right\|,\left\|\mathbf{h}_{2}\right\|\right) .
$$

We can easily show that (11) is equivalent to:

$$
\min _{\mathbf{s} \in \mathcal{S}^{2}}\left\|\mathbf{Q}^{H} \mathbf{y}-\mathbf{R} \Psi \mathbf{s}\right\|^{2} .
$$

Pre-multiplying (4) with $\mathbf{Q}^{H}$, we get

$$
\begin{aligned}
\mathbf{Q}^{H} \mathbf{y} & \triangleq \underbrace{\left[\begin{array}{c}
\bar{y}_{m} \\
\bar{y}_{m+5}
\end{array}\right]}_{\triangleq \mathbf{y}_{\text {equ }}} \\
& =\underbrace{\left[\begin{array}{cc}
\left\|\mathbf{h}_{1}\right\| & 0 \\
0 & \left\|\mathbf{h}_{2}\right\|
\end{array}\right]}_{\mathbf{R}}\left[\begin{array}{ll}
\psi_{11} & \psi_{12} \\
\psi_{21} & \psi_{22}
\end{array}\right]\left[\begin{array}{c}
s_{m} \\
s_{m+5}
\end{array}\right]+\left[\begin{array}{c}
\bar{w}_{m} \\
\bar{w}_{m+5}
\end{array}\right],
\end{aligned}
$$

where $\bar{w}_{m}$ and $\bar{w}_{m+5}$ are i.i.d. $\mathcal{C N}\left(0, N_{0}\right)$ since $\mathbf{Q}$ is semiunitary. Using the structure of $\mathbf{f}_{1}$ and $\mathbf{f}_{2}$, we can show that

$$
\left[\begin{array}{c}
\bar{y}_{m} \\
\bar{y}_{m+5}
\end{array}\right]=\left[\begin{array}{c}
\frac{\mathbf{h}_{1}^{H} \mathbf{y}_{m}}{\left\|\mathbf{h}_{1}\right\|} \\
\frac{\mathbf{h}_{2}^{H} \mathbf{y}_{m+5}}{\left\|\mathbf{h}_{2}\right\|}
\end{array}\right],
$$

and

$$
\left[\begin{array}{c}
\bar{w}_{m} \\
\bar{w}_{m+5}
\end{array}\right]=\left[\begin{array}{c}
\frac{\mathbf{h}_{1}^{H} \mathbf{w}_{m}}{\left\|\mathbf{h}_{1}\right\|} \\
\frac{\mathbf{h}_{2}^{H} \mathbf{w}_{m+5}}{\left\|\mathbf{h}_{2}\right\|}
\end{array}\right] \text {. }
$$

The interpretation is that we can apply MRC independently on all the subcarriers of symbols $m$ and $(m+5)$, and then 


$$
\begin{aligned}
\mathbb{E}_{\mathbf{h}_{\left\lceil\frac{m}{5}\right\rceil}\left[p\left(\mathbf{Y}_{m} \mid \mathbf{h}_{\left\lceil\frac{m}{5}\right\rceil}, s_{m}, \mathbf{d}_{p}, N_{0}\right)\right]}=\frac{1}{\left(\pi N_{0}\right)^{3 N_{\mathrm{sc}}^{\mathrm{RB}}}} \mathbb{E}_{\mathbf{h}_{\left\lceil\frac{m}{5}\right\rceil}}\left[\exp \left(-\frac{\left\|\mathbf{Y}_{m}-\mathbf{h}_{\left\lceil\frac{m}{5}\right\rceil} \mathbf{s}_{\mathrm{pm}}^{T}\right\|^{2}}{N_{0}}\right)\right] \\
=\frac{\operatorname{det}(\mathbf{U})}{\left(\pi N_{0}\right)^{3 N_{\mathrm{sc}}^{\mathrm{RB}}} \operatorname{det}\left(\mathbf{R}_{h h}\right)} \exp \left(\frac{-\operatorname{tr}\left(\mathbf{Y}_{m} \mathbf{Y}_{m}^{H}\right)}{N_{0}}+\frac{\mathbf{s}_{\mathrm{pm}}^{T} \mathbf{Y}_{m}^{H} \mathbf{U} \mathbf{Y}_{m} \mathbf{s}_{\mathrm{pm}}^{*}}{N_{0}^{2}}\right),
\end{aligned}
$$

perform joint detection with the system matrix $\mathbf{R} \Psi$. From (10), (14) and (15), we can write

$$
p\left(\mathbf{y} \mid \mathbf{F}, \mathbf{\Psi}, \mathbf{s}, N_{0}\right) \propto \frac{1}{\left(\pi N_{0}\right)^{2 N_{\mathrm{sc}}^{\mathrm{RB}}}} \exp \left(-\frac{\left\|\mathbf{y}_{\text {equ }}-\mathbf{R} \mathbf{\Psi} \mathbf{s}\right\|^{2}}{N_{0}}\right) .
$$

To compute the posterior LLR for the information bits that constitute $s_{k}$, we use:

$$
L\left(b_{k, i} \mid \mathbf{y}\right)=\log \left(\frac{\sum_{\mathbf{s}: b_{k, i}(\mathbf{s})=1} \exp \left(-\frac{\left\|\mathbf{y}_{\text {equ }}-\mathbf{R} \Psi \mathbf{s}\right\|^{2}}{N_{0}}\right)}{\sum_{\mathbf{s}: b_{k, i}(\mathbf{s})=0} \exp \left(-\frac{\left\|\mathbf{y}_{\text {equ }}-\mathbf{R} \Psi \mathbf{s}\right\|^{2}}{N_{0}}\right)}\right),
$$

where $\mathbf{s}: b_{k, i}(\mathbf{s})=\beta$ means all $\mathbf{s}$ for which the $i$ th bit of $s_{k}$ is equal to $\beta$. Note that here we are demodulating two symbols at a time, and each LLR computation involves the evaluation of 16 terms in (17). This is somewhat more complex than the conventional detection in Section IV-A.

\section{Optimal Non-COHERent Detectors}

In this section we consider the case where the receiver has only statistical knowledge about channel gains, and perfect knowledge about noise variance. Specifically, we assume that the receiver has knowledge about the distribution $p\left(\mathbf{h}_{l}\right), l=$ 1,2 and the value of $N_{0}$. One can view this case as the optimal non-coherent detection problem, which provides an upper bound on the performance when perfect CSI is not available at the receiver. In this case, received pilots are jointly processed with received payload.

\section{A. Conventional Coding Case}

Following the method described in [9], to derive the optimal non-coherent detection of $s_{m}, 1 \leq m \leq N_{\mathrm{symb}}^{\text {data }}$, we define $\mathbf{Y}_{m}$, which is a $N_{\mathrm{sc}}^{\mathrm{RB}} \times 3$ matrix defined as:

$$
\mathbf{Y}_{m}=\left[\begin{array}{ll}
\mathbf{Y}_{\left\lceil\frac{m}{5}\right\rceil}^{p} & \mathbf{y}_{m}
\end{array}\right]=\mathbf{h}_{\left\lceil\frac{m}{5}\right.} \underbrace{\left[\begin{array}{ll}
\mathbf{d}_{p}^{T} & s_{m}
\end{array}\right]}_{\triangleq \mathbf{s}_{\mathrm{pm}}^{T}}+\left[\begin{array}{ll}
\mathbf{W}_{l}^{p} & \mathbf{w}_{m}
\end{array}\right] .
$$

The optimal non-coherent detector for $s_{m}$ is obtained by maximizing the conditional probability $p\left(\mathbf{Y}_{m} \mid s_{m}, \mathbf{d}_{p}, N_{0}\right)$, which is the same as maximizing $\mathbb{E}_{\mathbf{h}_{\left\lceil\frac{m}{5}\right\rceil}}\left[p\left(\mathbf{Y}_{m} \mid \mathbf{h}_{\left\lceil\frac{m}{5}\right\rceil}, s_{m}, \mathbf{d}_{p}, N_{0}\right)\right]$, where

$$
\begin{aligned}
& p\left(\mathbf{Y}_{m} \mid \mathbf{h}_{\left\lceil\frac{m}{5}\right\rceil}, s_{m}, \mathbf{d}_{p}, N_{0}\right)=\frac{1}{\left(\pi N_{0}\right)^{3 N_{\mathrm{sc}}^{\mathrm{RB}}}} \times \\
& \exp \left(-\frac{\left\|\mathbf{Y}_{m}-\mathbf{h}_{\left\lceil\frac{m}{5}\right\rceil} \mathbf{s}_{\mathrm{pm}}^{T}\right\|^{2}}{N_{0}}\right) .
\end{aligned}
$$

Now suppose that $\mathbf{h}_{l} \sim \mathcal{C N}\left(\mathbf{0}, \mathbf{R}_{h h}\right)$, where $\mathbf{R}_{h h}$ is the covariance matrix of the channel gains on all $N_{\mathrm{sc}}^{\mathrm{RB}}$ subcarriers, i.e.,

$$
\mathbf{R}_{h h}=E\left(\mathbf{h}_{l} \mathbf{h}_{l}^{H}\right), \quad l=1,2 .
$$

Then we can simplify $\mathbb{E}_{\mathbf{h}_{\left\lceil\frac{m}{5}\right\rceil}}\left[p\left(\mathbf{Y}_{m} \mid \mathbf{h}_{\left\lceil\frac{m}{5}\right\rceil}, s_{m}, \mathbf{d}_{p}, N_{0}\right)\right]$ using standard techniques as shown in (21) on top of this page, where $\mathbf{U}=\left(\frac{\left\|\mathbf{s}_{\mathrm{pm}}\right\|^{2}}{N_{0}} \mathbf{I}+\mathbf{R}_{h h}^{-1}\right)^{-1} \cdot{ }^{10}$ Since all $s_{m}$ are QPSK symbols, $\operatorname{det}(\mathbf{U})$ is independent of $s_{m}$ (only a function of $\left|s_{m}\right|^{2}$ ). Hence, we can write the LLRs for the bits that constitute $s_{m}$ as:

$$
L\left(b_{i} \mid \mathbf{Y}_{m}\right)=\log \left(\frac{\sum_{s_{m}: b_{i}\left(s_{m}\right)=1} \exp \left(\frac{\mathbf{s}_{\mathrm{pm}}^{T} \mathbf{Y}_{m}^{H} \mathbf{U} \mathbf{Y}_{m} \mathbf{s}_{\mathrm{pm}}^{*}}{N_{0}^{2}}\right)}{\sum_{s_{m}: b_{i}\left(s_{m}\right)=0} \exp \left(\frac{\mathbf{s}_{\mathrm{pm}}^{T} \mathbf{Y}_{m}^{H} \mathbf{U} \mathbf{Y}_{m} \mathbf{s}_{\mathrm{pm}}^{*}}{N_{0}^{2}}\right)}\right) .
$$

\section{B. Proposed Precoding Case}

For this case, we write the combined received signal on the reference signal symbols as well as the data symbols $m$ and $m+5$ as:

$$
\begin{aligned}
& \underbrace{\left[\begin{array}{ccc}
\mathbf{y}_{11}^{p} & \mathbf{y}_{12}^{p} & \mathbf{y}_{m} \\
\mathbf{y}_{21}^{p} & \mathbf{y}_{22}^{p} & \mathbf{y}_{m+5}
\end{array}\right]}_{\triangleq \tilde{\mathbf{Y}}_{m}}=\left[\begin{array}{cc}
\mathbf{h}_{1} & \mathbf{0} \\
\mathbf{0} & \mathbf{h}_{2}
\end{array}\right]\left[\begin{array}{lll}
s_{p_{1}} & s_{p_{2}} & x_{m} \\
s_{p_{1}} & s_{p_{2}} & x_{m+5}
\end{array}\right]+ \\
& \underbrace{\left[\begin{array}{ccc}
\mathbf{w}_{11}^{p} & \mathbf{w}_{12}^{p} & \mathbf{w}_{m} \\
\mathbf{w}_{21}^{p} & \mathbf{w}_{22}^{p} & \mathbf{w}_{m+5}
\end{array}\right]}_{\triangleq \tilde{\mathbf{w}}_{m}}
\end{aligned}
$$

which can be rearranged as

$$
\underbrace{\operatorname{vec}\left(\tilde{\mathbf{Y}}_{m}\right)}_{\triangleq \mathbf{y}_{\mathrm{pm}}}=\underbrace{\left[\begin{array}{cc}
s_{p_{1}} \mathbf{I} & \mathbf{0} \\
\mathbf{0} & s_{p_{1}} \mathbf{I} \\
s_{p_{2}} \mathbf{I} & \mathbf{0} \\
\mathbf{0} & s_{p_{2}} \mathbf{I} \\
x_{m} \mathbf{I} & \mathbf{0} \\
\mathbf{0} & x_{m+5} \mathbf{I}
\end{array}\right]}_{\triangleq \tilde{\mathbf{X}}} \underbrace{\left[\begin{array}{l}
\mathbf{h}_{1} \\
\mathbf{h}_{2}
\end{array}\right]}_{\triangleq \mathbf{f}}+\operatorname{vec}\left(\tilde{\mathbf{W}}_{m}\right) \text {. }
$$

The optimal detector for $\mathbf{s}$ is obtained by maximizing the conditional distribution of $p\left(\mathbf{y}_{\mathrm{pm}} \mid \mathbf{s}, \mathbf{\Psi}, \mathbf{d}_{p}, N_{0}\right)$, which is same as maximizing $\mathbb{E}_{\mathbf{f}}\left[p\left(\mathbf{y}_{\mathrm{pm}} \mid \mathbf{f}, \mathbf{s}, \mathbf{\Psi}, \mathbf{d}_{p}, N_{0}\right)\right]$, where

$$
p\left(\mathbf{y}_{\mathrm{pm}} \mid \mathbf{f}, \mathbf{s}, \Psi, \mathbf{d}_{p}, N_{0}\right)=\frac{1}{\left(\pi N_{0}\right)^{6 N_{\mathrm{sc}}^{\mathrm{RB}}}} \exp \left(-\frac{\left\|\mathbf{y}_{\mathrm{pm}}-\tilde{\mathbf{X}} \mathbf{f}\right\|^{2}}{N_{0}}\right),
$$

\footnotetext{
${ }^{10}$ Note that since $\mathbf{R}_{h h}$ is the correlation matrix of the channel gains on successive subcarriers, it may be ill-conditioned. So we use $\mathbf{U}=$ $\mathbf{R}_{h h}\left(\frac{\left\|\mathbf{s}_{\mathrm{pm}}\right\|^{2}}{N_{0}} \mathbf{R}_{h h}+\mathbf{I}\right)^{-1}$.
} 
and $\mathbb{E}_{\mathbf{f}}\left[p\left(\mathbf{y}_{\mathrm{pm}} \mid \mathbf{f}, \mathbf{s}, \mathbf{\Psi}, \mathbf{d}_{p}, N_{0}\right)\right]$ can be simplified as:

$$
\begin{gathered}
\mathbb{E}_{\mathbf{f}}\left[p\left(\mathbf{y}_{\mathrm{pm}} \mid \mathbf{f}, \mathbf{s}, \mathbf{\Psi}, \mathbf{d}_{p}, N_{0}\right)\right]=\frac{\operatorname{det}\left(\mathbf{U}_{\mathrm{CFC}}\right)}{\left(\pi N_{0}\right)^{6 N_{\mathrm{sc}}^{\mathrm{RB}}} \operatorname{det}\left(\mathbf{R}_{\mathrm{CFC}}\right)} \times \\
\exp \left(-\frac{\left\|\mathbf{y}_{\mathrm{pm}}\right\|^{2}}{N_{0}}+\frac{\mathbf{y}_{\mathrm{pm}}^{H} \tilde{\mathbf{X}} \mathbf{U}_{\mathrm{CFC}} \tilde{\mathbf{X}}^{H} \mathbf{y}_{\mathrm{pm}}}{N_{0}^{2}}\right)
\end{gathered}
$$

where $\mathbf{R}_{\mathrm{CFC}}=\operatorname{diag}\left(\mathbf{R}_{h h}, \mathbf{R}_{h h}\right)$ and $\mathbf{U}_{\mathrm{CFC}}=$ $\left(\frac{\tilde{\mathbf{x}}^{H} \tilde{\mathbf{x}}}{N_{0}}+\mathbf{R}_{\mathrm{CFC}}^{-1}\right)^{-1}$. We can simplify $\mathbf{U}_{\mathrm{CFC}}$ as

$\mathbf{U}_{\mathrm{CFC}}=\left[\begin{array}{cc}\frac{\left\|\mathbf{d}_{p}\right\|^{2}+\left|x_{m}\right|^{2}}{N_{0}} \mathbf{I}+\mathbf{R}_{h h}^{-1} & \mathbf{0} \\ \mathbf{0} & \frac{\left\|\mathbf{d}_{p}\right\|^{2}+\left|x_{m+5}\right|^{2}}{N_{0}} \mathbf{I}+\mathbf{R}_{h h}^{-1}\end{array}\right]^{-1}$,

and we can easily note that for any pair of QPSK modulation symbols $s_{m}$ and $s_{m+5}$ in $\mathbf{s}$, and with the $\boldsymbol{\Psi}$ matrix specified in (5), $\left|x_{m}\right|^{2}$ and $\left|x_{m+5}\right|^{2}$ can take on only two possible values. This implies that $\operatorname{det}\left(\mathbf{U}_{\mathrm{CFC}}\right)$ is independent of $\mathbf{s}$. We can write the posterior LLRs for the information bits as:

$$
L\left(b_{k, i} \mid \mathbf{y}\right)=\log \left(\frac{\sum_{\mathbf{s}: b_{k, i}(\mathbf{s})=1} \exp \left(\frac{\mathbf{y}_{\mathrm{pm}}^{H} \tilde{\mathbf{X}} \mathbf{U}_{\mathrm{CFC}} \tilde{\mathbf{X}}^{H} \mathbf{y}_{\mathrm{pm}}}{N_{0}^{2}}\right)}{\sum_{\mathbf{s}: b_{k, i}(\mathbf{s})=0} \exp \left(\frac{\mathbf{y}_{\mathrm{pm}}^{H} \tilde{\mathbf{X}} \mathbf{U}_{\mathrm{CFC}} \tilde{\mathbf{X}}^{H} \mathbf{y}_{\mathrm{pm}}}{N_{0}^{2}}\right)}\right) .
$$

\section{Optimal Detectors with Channel Estimation AND KNOWN $N_{0}$}

In this section, we derive the optimal detectors when the receiver has an estimate of the channel gains obtained from the pilot symbols, but perfect knowledge of $N_{0}$. We consider LS and MMSE channel estimation methods in this work. Let the estimated channel on two slots be denoted as $\hat{\mathbf{h}}_{l, \mathrm{~L}}$ and $\hat{\mathbf{h}}_{l, \mathrm{M}}, \quad l=1,2$ for LS and MMSE estimation methods respectively. The detectors obtained by replacing $\mathbf{h}_{l}$ with $\hat{\mathbf{h}}_{l, \mathrm{~L}}$ or $\hat{\mathbf{h}}_{l, \mathrm{M}}$ in (7) and (11) will be referred to as "mismatched detectors".

\section{A. Conventional Coding Case}

1) LS Estimation: In both the time slots, using the reference signal symbols, we can obtain the LS estimate of the channel as:

$$
\begin{aligned}
\hat{\mathbf{h}}_{l, \mathrm{~L}} & =\arg \min _{\mathbf{h}_{l}}\left\|\mathbf{Y}_{l}^{p}-\mathbf{h}_{l} \mathbf{d}_{p}^{T}\right\|^{2}=\mathbf{Y}_{l}^{p} \mathbf{d}_{p}^{*}\left(\mathbf{d}_{p}^{T} \mathbf{d}_{p}^{*}\right)^{-1}=\frac{\mathbf{Y}_{l}^{p} \mathbf{d}_{p}^{*}}{\left\|\mathbf{d}_{p}\right\|^{2}} \\
& =\frac{\left(\mathbf{h}_{l} \mathbf{d}_{p}^{T}+\mathbf{W}_{l}^{p}\right) \mathbf{d}_{p}^{*}}{\left\|\mathbf{d}_{p}\right\|^{2}}=\mathbf{h}_{l}+\frac{\mathbf{W}_{l}^{p} \mathbf{d}_{p}^{*}}{\left\|\mathbf{d}_{p}\right\|^{2}}
\end{aligned}
$$

From (28), we can see that, with LS estimation of the channel, the estimation error $\boldsymbol{\delta}_{l, \mathrm{~L}} \triangleq \frac{\mathbf{W}_{l}^{p} \mathbf{d}_{p}^{*}}{\left\|\mathbf{d}_{p}\right\|^{2}}$ is independent of $\mathbf{h}_{l}$ and has the covariance matrix

$$
\mathbf{R}_{\delta \delta}^{\mathrm{LS}}=\frac{N_{0}}{\left\|\mathbf{d}_{p}\right\|^{2}} \mathbf{I} .
$$

From which we can write $\hat{\mathbf{h}}_{l, \mathrm{~L}} \sim \mathcal{C N}\left(\mathbf{0}, \mathbf{R}_{h h}+\mathbf{R}_{\delta \delta}^{\mathrm{LS}}\right)$.

We now derive the optimal detector with LS estimate of the channel gains by computing the conditional probability $p\left(\mathbf{y}_{m} \mid \hat{\mathbf{h}}_{\left\lceil\frac{m}{5}\right\rceil, \mathrm{L}}, s_{m}, N_{0}\right)$. We note that $\mathbf{y}_{m}, \hat{\mathbf{h}}_{\left\lceil\frac{m}{5}\right\rceil, \mathrm{L}}$ are jointly
Gaussian, conditioned on $s_{m}$ and $N_{0}$, with the following joint distribution:

$$
\left[\begin{array}{c}
\mathbf{y}_{m} \\
\hat{\mathbf{h}}_{\left\lceil\frac{m}{5}\right\rceil, \mathrm{L}}
\end{array}\right] \sim \mathcal{C N}\left(\mathbf{0},\left[\begin{array}{cc}
N_{0} \mathbf{I}+\left|s_{m}\right|^{2} \mathbf{R}_{h h} & \mathbf{R}_{h h} s_{m} \\
\mathbf{R}_{h h} s_{m}^{*} & \mathbf{R}_{h h}+\mathbf{R}_{\delta \delta}^{\mathrm{LS}}
\end{array}\right]\right)
$$

We can now write the following conditional distribution:

$$
\mathbf{y}_{m} \mid \hat{\mathbf{h}}_{\left\lceil\frac{m}{5}\right\rceil, \mathrm{L}}, s_{m}, N_{0} \sim \mathcal{C N}\left(\overline{\mathbf{y}}_{m, \mathrm{LS}}, \mathbf{P}_{\mathrm{LS}}\right)
$$

where [17],

$$
\begin{aligned}
\overline{\mathbf{y}}_{m, \mathrm{LS}} & \triangleq\left[\mathbf{R}_{h h}\left(\mathbf{R}_{h h}+\mathbf{R}_{\delta \delta}^{\mathrm{LS}}\right)^{-1}\right] \hat{\mathbf{h}}_{\left\lceil\frac{m}{5}\right\rceil, \mathrm{L}} s_{m}, \text { and } \\
\mathbf{P}_{\mathrm{LS}} & \triangleq\left(N_{0} \mathbf{I}+\left|s_{m}\right|^{2} \mathbf{R}_{h h}\left(\mathbf{I}-\left(\mathbf{R}_{h h}+\mathbf{R}_{\delta \delta}^{\mathrm{LS}}\right)^{-1} \mathbf{R}_{h h}\right)\right) .
\end{aligned}
$$

Hence we can write

$$
\begin{aligned}
p\left(\mathbf{y}_{m} \mid \hat{\mathbf{h}}_{\left\lceil\frac{m}{5}\right\rceil, \mathrm{L}}, s_{m}, N_{0}\right)=\frac{1}{\pi^{N_{\mathrm{sc}}^{\mathrm{RB}}} \operatorname{det}\left(\mathbf{P}_{\mathrm{LS}}\right)} \times \\
\quad \exp \left(-\left(\mathbf{y}_{m}-\overline{\mathbf{y}}_{m, \mathrm{LS}}\right)^{H} \mathbf{P}_{\mathrm{LS}}^{-1}\left(\mathbf{y}_{m}-\overline{\mathbf{y}}_{m, \mathrm{LS}}\right)\right) .
\end{aligned}
$$

Using $\mathbf{P}_{\mathrm{LS}}^{-1}=\mathbf{P}_{\mathrm{LS}}^{-1 / 2} \mathbf{P}_{\mathrm{LS}}^{-1 / 2}$, where $\mathbf{P}_{\mathrm{LS}}^{-1 / 2}$ is a positive definite square-root of $\mathbf{P}_{\mathrm{LS}}^{-1}$, we can rewrite (34) as

$$
\begin{aligned}
p\left(\mathbf{y}_{m} \mid \hat{\mathbf{h}}_{\left\lceil\frac{m}{5}\right\rceil, \mathrm{L}}, s_{m}, N_{0}\right) & =\frac{1}{\pi^{N_{\mathrm{sc}}^{\mathrm{RB}}} \operatorname{det}\left(\mathbf{P}_{\mathrm{LS}}\right)} \times \\
& \exp \left(-\left\|\tilde{\mathbf{y}}_{m, \mathrm{LS}}-\check{\mathbf{y}}_{m, \mathrm{LS}}\right\|^{2}\right),
\end{aligned}
$$

where

$$
\tilde{\mathbf{y}}_{m, \mathrm{LS}} \triangleq \mathbf{P}_{\mathrm{LS}}^{-1 / 2} \mathbf{y}_{m} \text { and } \check{\mathbf{y}}_{m, \mathrm{LS}} \triangleq \mathbf{P}_{\mathrm{LS}}^{-1 / 2} \overline{\mathbf{y}}_{m, \mathrm{LS}} .
$$

Using (35), with LS estimation of the channel gains, we can write the LLRs for the bits that constitute $s_{m}$ as:

$L\left(b_{i} \mid \mathbf{y}_{m}\right)=\log \left(\frac{\sum_{s_{m}: b_{i}\left(s_{m}\right)=1} \exp \left(-\left\|\tilde{\mathbf{y}}_{m, \mathrm{LS}}-\check{\mathbf{y}}_{m, \mathrm{LS}}\right\|^{2}\right)}{\sum_{s_{m}: b_{i}\left(s_{m}\right)=0} \exp \left(-\left\|\tilde{\mathbf{y}}_{m, \mathrm{LS}}-\check{\mathbf{y}}_{m, \mathrm{LS}}\right\|^{2}\right)}\right)$

2) MMSE Estimation: To obtain the MMSE channel estimator for $\mathbf{h}_{l}$, we rewrite the received signal on both the reference signal symbols in a time slot as

$$
\underbrace{\operatorname{vec}\left(\mathbf{Y}_{l}^{p}\right)}_{\triangleq \mathbf{y}_{p}}=\underbrace{\left(\mathbf{d}_{p} \otimes \mathbf{I}\right)}_{\triangleq \mathbf{P}} \mathbf{h}_{l}+\underbrace{\operatorname{vec}\left(\mathbf{W}_{l}^{p}\right)}_{\triangleq \mathbf{w}_{p}}
$$

and we define

$$
\hat{\mathbf{h}}_{l, \mathrm{M}} \triangleq \mathbf{G}_{\mathrm{MMSE}} \mathbf{y}_{p}
$$

with

$$
\mathbf{G}_{\mathrm{MMSE}}=\arg \min _{\mathbf{G}} \mathbb{E}\left[\left\|\mathbf{h}_{l}-\mathbf{G y}_{p}\right\|^{2}\right]=\mathbf{R}_{h y_{p}} \mathbf{R}_{y_{p} y_{p}}^{-1},
$$

where $\mathbf{R}_{h y_{p}}=\mathbb{E}\left[\mathbf{h}_{l} \mathbf{y}_{p}^{H}\right]=\mathbf{R}_{h h} \mathbf{P}^{H}$ and $\mathbf{R}_{y_{p} y_{p}}=$ $\mathbb{E}\left[\mathbf{y}_{p} \mathbf{y}_{p}^{H}\right]=\mathbf{P R}_{h h} \mathbf{P}^{H}+N_{0} \mathbf{I}$. While the LS estimator does not need any additional information about the channel statistics, MMSE estimator needs information about $\mathbf{R}_{h h}$ and 
$N_{0}$. We can easily show that the covariance matrix of the estimation error with the MMSE estimation is given by

$$
\begin{aligned}
\mathbf{R}_{\delta \delta}^{\mathrm{MMSE}} & =\mathbb{E}\left[\left(\hat{\mathbf{h}}_{l, \mathrm{M}}-\mathbf{h}_{l}\right)\left(\hat{\mathbf{h}}_{l, \mathrm{M}}-\mathbf{h}_{l}\right)^{H}\right] \\
& =\left(\mathbf{R}_{h h}^{-1}+\frac{\left\|\mathbf{d}_{p}\right\|^{2}}{N_{0}} \mathbf{I}\right)^{-1} .
\end{aligned}
$$

Note that, for the case of MMSE estimation as well we can write

$$
\hat{\mathbf{h}}_{l, \mathrm{M}}=\mathbf{h}_{l}+\boldsymbol{\delta}_{l, \mathrm{M}},
$$

however, $\mathbf{h}_{l}$ and $\boldsymbol{\delta}_{l, \mathrm{M}}$ are not independent as in the case of LS estimation. ${ }^{11}$ On the $m$ th data symbol, we can write

$$
\begin{aligned}
\mathbf{y}_{m} & =\mathbf{h}_{\left\lceil\frac{m}{5}\right\rceil} s_{m}+\mathbf{w}_{m} \\
& =\hat{\mathbf{h}}_{\left\lceil\frac{m}{5}\right\rceil, M} s_{m}-\underbrace{\boldsymbol{\delta}_{\left\lceil\frac{m}{5}\right\rceil, M} s_{m}+\mathbf{e}_{m}}_{\triangleq \mathbf{n}_{m}}
\end{aligned}
$$

with $\mathbf{n}_{m}$ and $\hat{\mathbf{h}}_{\left\lceil\frac{m}{5}\right\rceil, M}$ being independent, we can write

$$
\begin{aligned}
& p\left(\mathbf{y}_{m} \mid \hat{\mathbf{h}}_{\left\lceil\frac{m}{5}\right\rceil, \mathrm{M}}, s_{m}, N_{0}\right)=\frac{1}{\pi^{N_{\mathrm{sc}}^{\mathrm{RB}}} \operatorname{det}\left(\mathbf{P}_{\mathrm{MMSE}}\right)} \times \\
& \exp \left(-\left(\mathbf{y}_{m}-\hat{\mathbf{h}}_{\left\lceil\frac{m}{5}\right\rceil, \mathrm{M}} s_{m}\right)^{H} \mathbf{P}_{\mathrm{MMSE}}^{-1}\left(\mathbf{y}_{m}-\hat{\mathbf{h}}_{\left\lceil\frac{m}{5}\right\rceil, \mathrm{M}} s_{m}\right)\right),
\end{aligned}
$$

where $\mathbf{P}_{\mathrm{MMSE}} \triangleq N_{0} \mathbf{I}+\mathbf{R}_{\delta \delta}^{\mathrm{MMSE}}\left|s_{m}\right|^{2}$. We can equivalently write (43) as:

$$
\begin{aligned}
& p\left(\mathbf{y}_{m} \mid \hat{\mathbf{h}}_{\left\lceil\frac{m}{5}\right\rceil, \mathrm{M}}, s_{m}, N_{0}\right)=\frac{1}{\pi^{N_{\mathrm{sc}}^{\mathrm{RB}}} \operatorname{det}\left(\mathbf{P}_{\mathrm{MMSE}}\right)} \times \\
& \quad \exp \left(-\left\|\tilde{\mathbf{y}}_{m, \mathrm{MMSE}}-\check{\mathbf{y}}_{m, \mathrm{MMSE}}\right\|^{2}\right),
\end{aligned}
$$

where

$$
\begin{aligned}
& \tilde{\mathbf{y}}_{m, \mathrm{MMSE}} \triangleq \mathbf{P}_{\mathrm{MMSE}}^{-1 / 2} \mathbf{y}_{m}, \text { and } \\
& \check{\mathbf{y}}_{m, \mathrm{MMSE}} \triangleq \mathbf{P}_{\mathrm{MMSE}}^{-1 / 2} \hat{\mathbf{h}}_{\left\lceil\frac{m}{5}\right\rceil, \mathrm{M}} s_{m} .
\end{aligned}
$$

Using (44), with MMSE estimation of the channel gains, we can write the LLRs for the bits that constitute $s_{m}$ as:

$$
\begin{aligned}
& L\left(b_{i} \mid \mathbf{y}_{m}\right)= \\
& \log \left(\frac{\sum_{s_{m}: b_{i}\left(s_{m}\right)=1} \exp \left(-\left\|\tilde{\mathbf{y}}_{m, \mathrm{MMSE}}-\check{\mathbf{y}}_{m, \mathrm{MMSE}}\right\|^{2}\right)}{\sum_{s_{m}: b_{i}\left(s_{m}\right)=0} \exp \left(-\left\|\tilde{\mathbf{y}}_{m, \mathrm{MMSE}}-\check{\mathbf{y}}_{m, \mathrm{MMSE}}\right\|^{2}\right)}\right) .
\end{aligned}
$$

\section{B. Proposed Precoding Case}

1) LS Estimation: The combined received signal vector in (4) can equivalently be written as

$$
\mathbf{y}=\mathbf{X f}+\mathbf{w}
$$

where $\mathbf{X}=\operatorname{diag}\left(x_{m} \mathbf{I}, x_{m+5} \mathbf{I}\right)$ and $\mathbf{f}=\left[\begin{array}{ll}\mathbf{h}_{1}^{T} & \mathbf{h}_{2}^{T}\end{array}\right]^{T}$. We can write the LS estimate of $\mathbf{f}$ as $\hat{\mathbf{f}}_{\mathrm{L}}=\mathbf{f}+\boldsymbol{\delta}_{\mathrm{L}}$, where $\boldsymbol{\delta}_{\mathrm{L}}=\left[\begin{array}{ll}\boldsymbol{\delta}_{1, \mathrm{~L}}^{T} & \boldsymbol{\delta}_{2, \mathrm{~L}}^{T}\end{array}\right]^{T}$ denote the corresponding estimation error with $\boldsymbol{\delta}_{1, \mathrm{~L}}$ and $\boldsymbol{\delta}_{2, \mathrm{~L}}$ being independent. Then $\mathbf{y}$ and $\hat{\mathbf{f}}_{\mathrm{L}}$

\footnotetext{
${ }^{11} \hat{\mathbf{h}}_{l, \mathrm{M}}$ and $\boldsymbol{\delta}_{l, \mathrm{M}}$ are independent.
}

are jointly Gaussian conditioned on $\mathbf{x}$ and $N_{0}$, and the joint conditional distribution is given by:

$$
\left[\begin{array}{c}
\mathbf{y} \\
\hat{\mathbf{f}}_{\mathrm{L}}
\end{array}\right] \sim \mathcal{C N}\left(\mathbf{0},\left[\begin{array}{cc}
\mathbf{A} & \mathbf{B} \\
\mathbf{B}^{H} & \mathbf{D}
\end{array}\right]\right)
$$

where

$$
\begin{aligned}
& \mathbf{A}=\operatorname{diag}\left(\left|x_{m}\right|^{2} \mathbf{R}_{h h}+N_{0} \mathbf{I},\left|x_{m+5}\right|^{2} \mathbf{R}_{h h}+N_{0} \mathbf{I}\right) \\
& \mathbf{B}=\operatorname{diag}\left(x_{m} \mathbf{R}_{h h}, x_{m+5} \mathbf{R}_{h h}\right) \\
& \mathbf{D}=\operatorname{diag}\left(\mathbf{R}_{h h}+\mathbf{R}_{\delta \delta}^{\mathrm{LS}}, \mathbf{R}_{h h}+\mathbf{R}_{\delta \delta}^{\mathrm{LS}}\right)
\end{aligned}
$$

Using (47), we can write the conditional distribution of $\mathbf{y} \mid \hat{\mathbf{f}}_{\mathrm{L}}, \mathbf{\Psi}, \mathbf{s}, N_{0}$ as

$$
\begin{aligned}
& p\left(\mathbf{y} \mid \hat{\mathbf{f}}_{\mathrm{L}}, \boldsymbol{\Psi}, \mathbf{s}, N_{0}\right)=\frac{1}{\pi^{2 N_{\mathrm{sc}}^{\mathrm{RB}}} \operatorname{det}\left(\mathbf{T}_{\mathrm{LS}}\right)} \times \\
& \exp \left(-\left(\mathbf{y}-\overline{\mathbf{y}}_{\mathrm{LS}}\right)^{H} \mathbf{T}_{\mathrm{LS}}{ }^{-1}\left(\mathbf{y}-\overline{\mathbf{y}}_{\mathrm{LS}}\right)\right)
\end{aligned}
$$

where

$$
\overline{\mathbf{y}}_{\mathrm{LS}} \triangleq \mathbf{R}_{\mathrm{CFC}} \mathbf{D}^{-1} \hat{\mathbf{F}}_{\mathrm{L}} \boldsymbol{\Psi}_{\mathbf{S}}
$$

and $\hat{\mathbf{F}}_{\mathrm{L}}$ is defined similarly to $\mathbf{F}$ in (4) with $\mathbf{h}_{l}$ replaced by $\hat{\mathbf{h}}_{l, \mathrm{~L}}$ and $\mathbf{T}_{\mathrm{LS}} \triangleq \operatorname{diag}\left(\mathbf{P}_{m, \mathrm{LS}}, \mathbf{P}_{m+5, \mathrm{LS}}\right)$ with

$$
\begin{gathered}
\mathbf{P}_{m, \mathrm{LS}} \triangleq N_{0} \mathbf{I}+\left|x_{m}\right|^{2} \mathbf{R}_{h h}\left(\mathbf{I}-\left(\mathbf{R}_{h h}+\mathbf{R}_{\delta \delta}^{\mathrm{LS}}\right)^{-1} \mathbf{R}_{h h}\right), \\
\mathbf{P}_{m+5, \mathrm{LS}} \triangleq N_{0} \mathbf{I}+\left|x_{m+5}\right|^{2} \mathbf{R}_{h h}\left(\mathbf{I}-\left(\mathbf{R}_{h h}+\mathbf{R}_{\delta \delta}^{\mathrm{LS}}\right)^{-1} \mathbf{R}_{h h}\right) .
\end{gathered}
$$

The conditional distribution in (48) can equivalently be written as:

$p\left(\mathbf{y} \mid \hat{\mathbf{f}}_{\mathrm{L}}, \mathbf{\Psi}, \mathbf{s}, N_{0}\right)=\frac{1}{\pi^{2 N_{\mathrm{sc}}^{\mathrm{RB}}} \operatorname{det}\left(\mathbf{T}_{\mathrm{LS}}\right)} \exp \left(-\left\|\tilde{\mathbf{y}}_{\mathrm{LS}}-\check{\mathbf{y}}_{\mathrm{LS}}\right\|^{2}\right)$,

where

$$
\tilde{\mathbf{y}}_{\mathrm{LS}} \triangleq \mathbf{T}_{\mathrm{LS}}{ }^{-1 / 2} \mathbf{y}, \text { and } \check{\mathbf{y}}_{\mathrm{LS}} \triangleq \mathbf{T}_{\mathrm{LS}}{ }^{-1 / 2} \overline{\mathbf{y}}_{\mathrm{LS}}
$$

Using (50), for the proposed method with LS estimate of the channel gains, we can write the posterior LLRs for the information bits as:

$$
L\left(b_{k, i} \mid \mathbf{y}\right)=\log \left(\frac{\sum_{\mathbf{s}: b_{k, i}(\mathbf{s})=1} \exp \left(-\left\|\tilde{\mathbf{y}}_{\mathrm{LS}}-\check{\mathbf{y}}_{\mathrm{LS}}\right\|^{2}\right)}{\sum_{\mathbf{s}: b_{k, i}(\mathbf{s})=0} \exp \left(-\left\|\tilde{\mathbf{y}}_{\mathrm{LS}}-\check{\mathbf{y}}_{\mathrm{LS}}\right\|^{2}\right)}\right) .
$$

2) MMSE Estimation: Using the received signal model in (46), we can easily extend the steps described in Section VI-A2 and write the conditional distribution of $\mathbf{y} \mid \hat{\mathbf{f}}_{\mathrm{M}}, \Psi, \mathbf{s}, N_{0}$ as:

$$
\begin{aligned}
& p\left(\mathbf{y} \mid \hat{\mathbf{f}}_{\mathrm{M}}, \boldsymbol{\Psi}, \mathbf{s}, N_{0}\right)=\frac{1}{\pi^{2 N_{\mathrm{sc}}^{\mathrm{RB}}} \operatorname{det}\left(\mathbf{T}_{\mathrm{MMSE}}\right)} \times \\
& \quad \exp \left(-\left(\mathbf{y}-\mathbf{X} \hat{\mathbf{f}}_{\mathrm{M}}\right)^{H} \mathbf{T}_{\mathrm{MMSE}}{ }^{-1}\left(\mathbf{y}-\mathbf{X} \hat{\mathbf{f}}_{\mathrm{M}}\right)\right)
\end{aligned}
$$

where $\mathbf{T}_{\mathrm{MMSE}} \triangleq \operatorname{diag}\left(\mathbf{P}_{m, \mathrm{MMSE}}, \mathbf{P}_{m+5, \mathrm{MMSE}}\right)$ with

$$
\begin{gathered}
\mathbf{P}_{m, \mathrm{MMSE}} \triangleq N_{0} \mathbf{I}+\left|x_{m}\right|^{2} \mathbf{R}_{\delta \delta}^{\mathrm{MMSE}}, \\
\mathbf{P}_{m+5, \mathrm{MMSE}} \triangleq N_{0} \mathbf{I}+\left|x_{m+5}\right|^{2} \mathbf{R}_{\delta \delta}^{\mathrm{MMSE}} .
\end{gathered}
$$


The conditional distribution in (52) can equivalently be written as:

$$
\begin{gathered}
p\left(\mathbf{y} \mid \hat{\mathbf{f}}_{\mathrm{M}}, \mathbf{\Psi}, \mathbf{s}, N_{0}\right)=\frac{1}{\pi^{2 N_{\mathrm{sc}}^{\mathrm{RB}}} \operatorname{det}\left(\mathbf{T}_{\mathrm{MMSE}}\right)} \times \\
\exp \left(-\left\|\tilde{\mathbf{y}}_{\mathrm{MMSE}}-\check{\mathbf{y}}_{\mathrm{MMSE}}\right\|^{2}\right),
\end{gathered}
$$

where

$$
\tilde{\mathbf{y}}_{\mathrm{MMSE}} \triangleq \mathbf{T}_{\mathrm{MMSE}}{ }^{-1 / 2} \mathbf{y} \text { and } \check{\mathbf{y}}_{\mathrm{MMSE}} \triangleq \mathbf{T}_{\mathrm{MMSE}}{ }^{-1 / 2} \mathbf{X} \hat{\mathbf{f}}_{\mathrm{M}} \text {. }
$$

Using (53), in case of the proposed method with MMSE channel estimation, we can write the posterior LLRs for the information bits as: ${ }^{12}$

$$
L\left(b_{k, i} \mid \mathbf{y}\right)=\log \left(\frac{\sum_{\mathbf{s}: b_{k, i}(\mathbf{s})=1} \exp \left(-\left\|\tilde{\mathbf{y}}_{\mathrm{MMSE}}-\check{\mathbf{y}}_{\mathrm{MMSE}}\right\|^{2}\right)}{\sum_{\mathbf{s}: b_{k, i}(\mathbf{s})=0} \exp \left(-\left\|\tilde{\mathbf{y}}_{\mathrm{MMSE}}-\check{\mathbf{y}}_{\mathrm{MMSE}}\right\|^{2}\right)}\right) .
$$

\section{Optimal Detectors with Channel Estimation AND UNKNOWN $N_{0}$}

In practical systems like LTE, receivers do not have perfect knowledge of the noise variance. They need to estimate this quantity before detecting the modulated data. Towards this, we first estimate the channel using the LS estimator described in (28) and then use this estimated channel to estimate the noise variance value $N_{0}$ from the reference symbols. ${ }^{13}$ Using $\hat{\mathbf{h}}_{l, \mathrm{~L}}$, the maximum-likelihood (ML) estimate of the noise variance can be expressed as:

$$
\hat{N}_{0}=\frac{\left\|\mathbf{Y}_{1}^{p}-\hat{\mathbf{h}}_{1, \mathrm{~L}} \mathbf{d}_{p}^{T}\right\|^{2}+\left\|\mathbf{Y}_{2}^{p}-\hat{\mathbf{h}}_{2, \mathrm{~L}} \mathbf{d}_{p}^{T}\right\|^{2}}{4 N_{\mathrm{sc}}^{\mathrm{RB}}} .
$$

The estimated $\hat{N}_{0}$ value is then used for computing the LLR values in (36) and (51). Even though we could consider the case of unknown $N_{0}$ with non-coherent detectors described in Section V as well, marginalizing (21) and (26) over the distribution of $N_{0}$ results in computationally intractable problem, hence we do not consider this case in this work.

\section{Simulation Results}

In this section, we present simulation results to illustrate the performance of the proposed precoding method together with the performance of the conventional method for the different cases of CSI and noise variance knowledge availability at the receiver, as summarized in Table II. Monte-Carlo simulation was used to obtain the block-error rate (BLER) performance, and at each point in the curves, we observed at least 1000 block errors. We considered an OFDM system with 300 subcarriers (25 resource blocks) and a subcarrier spacing of $15 \mathrm{kHz}$. These parameters correspond to an LTE system with $5 \mathrm{MHz}$ channel BW [18]. For the fading process, we used the ITU - Vehicular A channel model with Rayleigh fading distribution [19]. We used normal CP configuration with $N_{I}=11$ and extended CP

\footnotetext{
${ }^{12}$ Note that $\operatorname{det}\left(\mathbf{T}_{\mathrm{MMSE}}\right)$ is independent of entries in $\mathbf{s}$.

${ }^{13} \mathrm{We}$ did not use MMSE channel estimate as it needs the knowledge of $N_{0}$ as can be seen from (39).
}

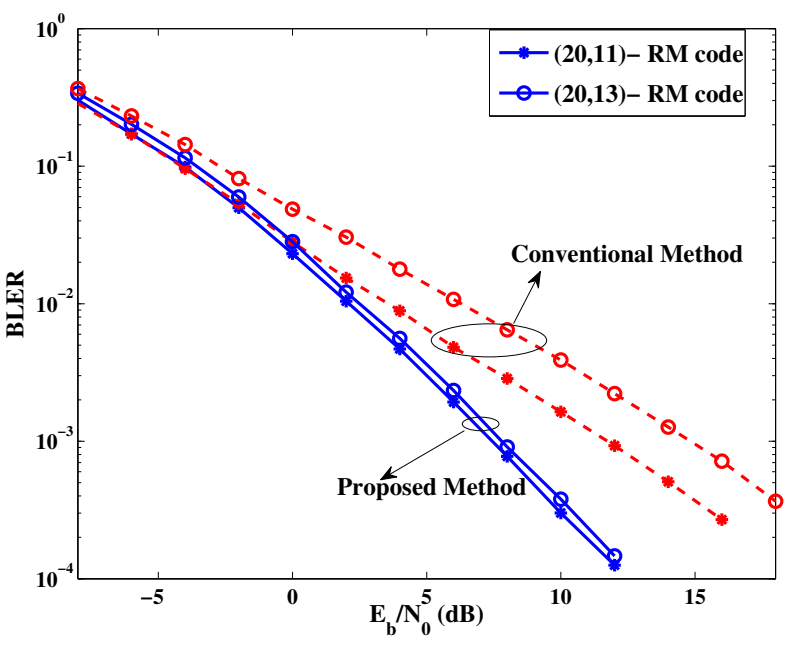

Figure 3. Performance comparison of the proposed method and the conventional coding method for both (20,11)-Reed-Muller code and $(20,13)$ Reed-Muller code. Here the channel gains and the value of $N_{0}$ is assumed to be perfectly known at the receiver. Both the transmitter and receiver are equipped with single antenna.

configuration for $N_{I}=13 .{ }^{14}$ We used a pseudo-random bit interleaver and de-interleaver pair, independently chosen for each Monte-Carlo run.

\section{A. Results with P-CSI and Known $N_{0}$ at the Receiver}

First we show the Reed-Muller code performance with perfect CSI and perfect knowledge of $N_{0}$ at the receiver. For soft-decoding of Reed-Muller code, we used an algorithm based on Hadamard matrices as described in [16]. Fig. 3 shows the performance comparison for the conventional coding and for the proposed method with soft-decision decoding. We see that the proposed method is performing better than the conventional coding method, both for $N_{I}=11$ and 13 . With P-CSI at the receiver and at a BLER of $10^{-3}$, the proposed method has a performance gain of up to $4.3 \mathrm{~dB}$ and $7 \mathrm{~dB}$ over the conventional coding method for $N_{I}=11$ and 13 , respectively. One can also note that, relative to $N_{I}=11$ bits, $N_{I}=13$ bits has more degradation in performance for the conventional method. The gain for the proposed method comes from the complex-field spreading of the information over the two independent frequency slots and there by extracting the available frequency diversity in a better way. The difference in the diversity order for the proposed method and the conventional method can easily be seen from the slope of the curves in the figure.

\section{B. Results with Channel Estimation and Known $N_{0}$ at the Receiver}

Next, we illustrate the performance when the receiver does not have P-CSI. Table II summarizes the various detectors considered. First we present the results for different types of

\footnotetext{
${ }^{14}$ Two reference signal symbols for normal $\mathrm{CP}$ and one reference signal symbol for extended CP configuration are available in each time slot to estimate the channel.
} 
Table II

SUMMARY OF VARIOUS CSI AND DETECTOR COMBINATIONS CONSIDERED IN THIS WORK

\begin{tabular}{|c|c|c|}
\hline Detector type & Conventional method & Proposed method \\
\hline Optimal P-CSI detector & $\begin{array}{c}\text { CSI: } \mathbf{h}_{l}, l=1,2 \\
\text { LLR: }(9) \\
N_{0}: \text { Known }\end{array}$ & $\begin{array}{c}\text { CSI: f } \\
\text { LLR: (17) } \\
N_{0}: \text { Known }\end{array}$ \\
\hline Optimal non-coherent detector & $\begin{array}{c}\text { CSI: Distribution of } \mathbf{h}_{l}, l=1,2 \\
\text { LLR: }(22) \\
N_{0}: \text { Known }\end{array}$ & $\begin{array}{l}\text { CSI: Distribution of } \mathbf{f} \\
\text { LLR: (27) } \\
N_{0}: \text { Known }\end{array}$ \\
\hline Mismatched detector & $\begin{array}{c}\text { CSI: } \hat{\mathbf{h}}_{l, \mathrm{~L}} \text { or } \hat{\mathbf{h}}_{l, \mathrm{M}}, l=1,2 \\
\text { LLR: }(9) \\
N_{0}: \begin{array}{l}\text { Known for MMSE, known } \\
\text { or unknown for LS }\end{array}\end{array}$ & $\begin{array}{c}\text { CSI: } \hat{\mathbf{f}}_{\mathrm{L}} \text { or } \hat{\mathbf{f}}_{\mathrm{M}} \\
\text { LLR: }(17) \\
N_{0}: \text { Known for MMSE, known } \\
\text { or unknown for LS }\end{array}$ \\
\hline $\begin{array}{c}\text { Optimal detector with channel } \\
\text { estimation }\end{array}$ & $\begin{aligned} \text { CSI: } \hat{\mathbf{h}}_{l, \mathrm{~L}} \text { or } \hat{\mathbf{h}}_{l, \mathrm{M}}, l=1,2 \\
\text { LLR: (36) or }(45) \\
N_{0}: \text { Known for MMSE, known } \\
\text { or unknown for LS }\end{aligned}$ & $\begin{array}{c}\text { CSI: } \hat{\mathbf{f}}_{\mathrm{L}} \text { or } \hat{\mathbf{f}}_{\mathrm{M}} \\
\text { LLR: }(51) \text { or }(54) \\
N_{0}: \text { Known for MMSE, known } \\
\text { or unknown for LS }\end{array}$ \\
\hline
\end{tabular}

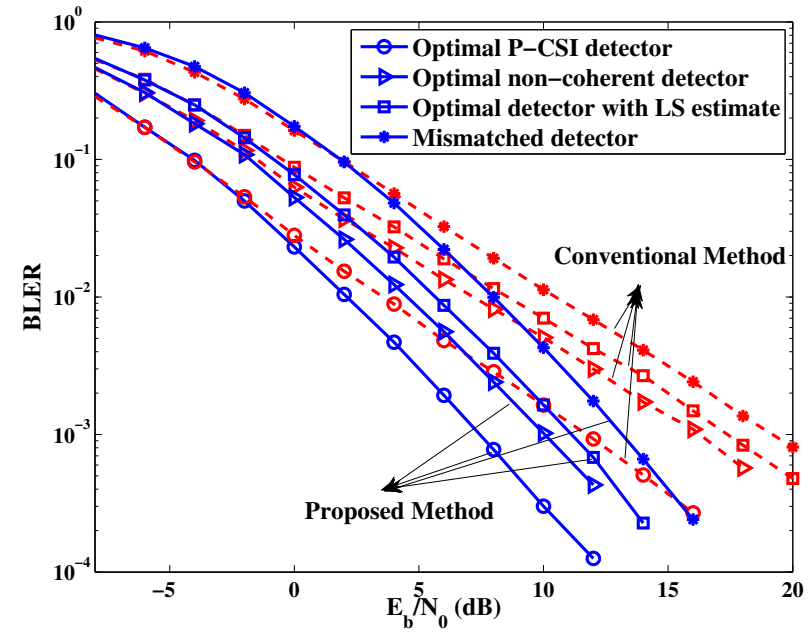

(a) LS estimate, $(20,11)$ - Reed-Muller code

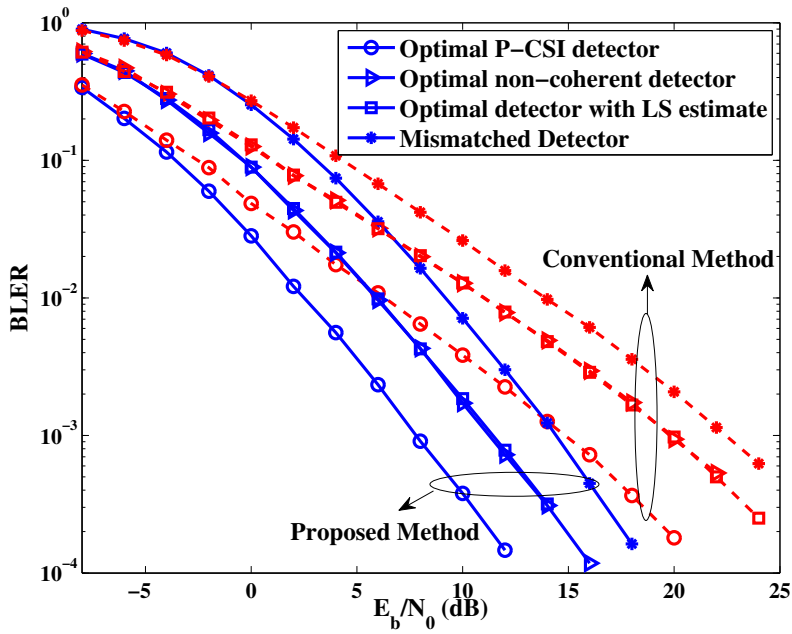

(b) LS estimate, $(20,13)$ - Reed-Muller code.

Figure 4. Comparison of performance for the various cases summarized in Table II. $N_{0}$ is assumed to be perfectly known at the receiver. We used LS estimation for estimating the channel. For comparison, we also show the performance of the non-coherent detectors. Both the transmitter and receiver are equipped with single antenna.

detectors with the receiver having perfect knowledge of $N_{0}$ value. For the case with channel estimation, we used LS and MMSE channel estimation methods.

Fig. 4 illustrates the performance comparison of the proposed method with the conventional method for the case of LS channel estimate. For reference purpose, we plotted results with P-CSI and optimal non-coherent detectors as well. We can see from Figs. 4(a) and 4(b) that mismatched detectors with a LS channel estimate have the poorest performance among the various cases considered in the study. By using the optimal detector for the LS estimate, one can reach close to the performance of the optimal non-coherent detectors. For all the detectors considered, the proposed method provides significant gains over the conventional method.

Fig. 5 illustrates the performance comparison of the proposed method with the conventional method for the case of MMSE channel estimation. One interesting observation from Figs. 5(a) and 5(b) is that the mismatched detectors with
MMSE channel estimation have performance similar to that of the optimal non-coherent detectors. In this case as well, detectors for the proposed method have significant gains over the corresponding detectors for the conventional method.

\section{Result with Unknown $N_{0}$}

Fig. 6 shows the performance comparison of the mismatched detector and the optimal detector for LS channel estimation with and without the knowledge of $N_{0}$ at the receiver. For estimating the noise power value, we used (55) with the LS estimate. As we can see, the unknown $N_{0}$ case has performance similar to the known $N_{0}$ case for both the proposed method and the conventional method.

\section{Result with Two Receive Antennas}

Fig. 7 shows the performance results for the case of 2 receive antennas. At a BLER of $10^{-3}$, with P-CSI at the receiver, the gain for the proposed method over the conventional method 


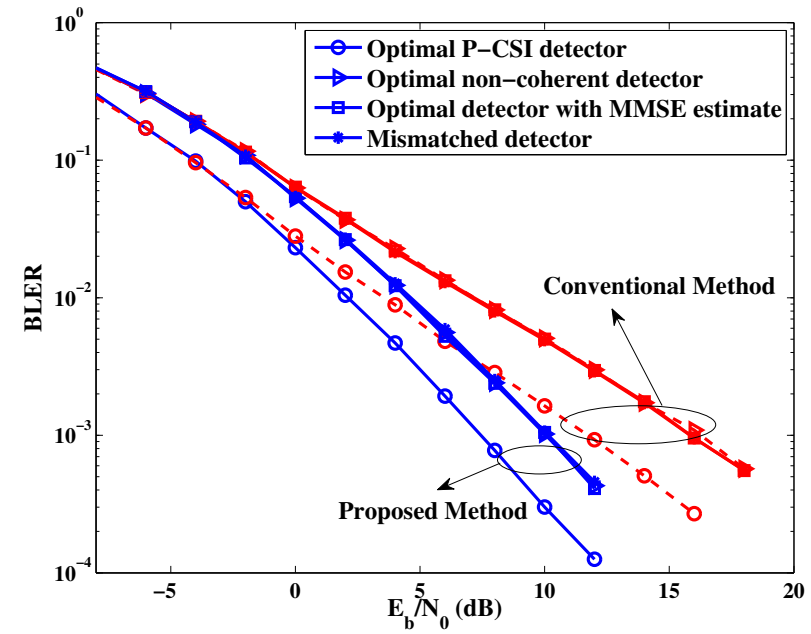

(a) MMSE estimate, $(20,11)$ - Reed-Muller code.

Figure 5. Same as Fig. 4 but with MMSE channel estimation.

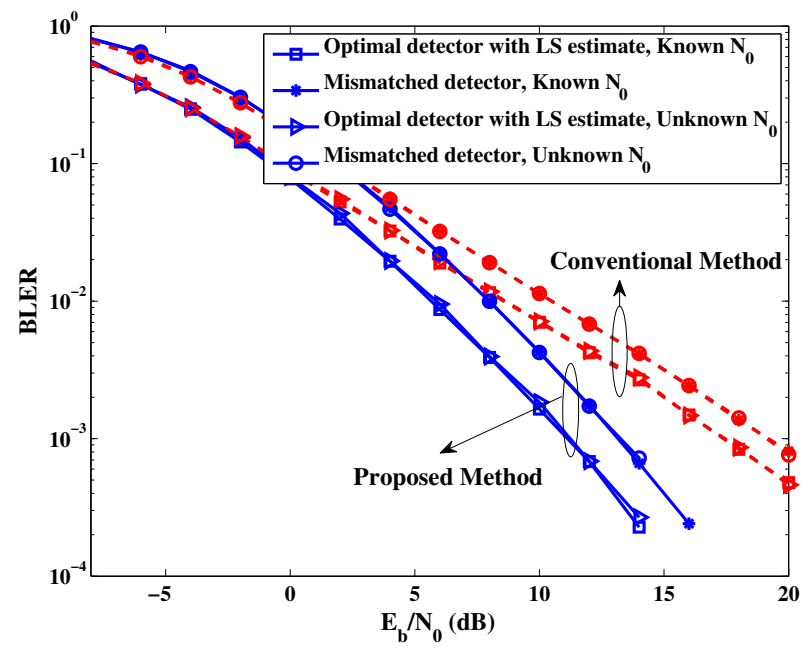

Figure 6. Performance comparison of the proposed method and the conventional coding method for the case when $N_{0}$ value is known and unknown at the receiver. Here we used the $(20,13)$-Reed-Muller code with LS channel estimation. Both the transmitter and receiver are equipped with single antenna.

is about $2 \mathrm{~dB}$. With MMSE channel estimation, the gain for the proposed method is about $3 \mathrm{~dB}$. The performance advantage of the proposed method relative to the conventional method is smaller in the multiple-antenna case. In other words, additional receive antennas help the conventional method more than they help the proposed method. The reason for this is that the ReedMuller code is reasonably powerful for AWGN channels, but not especially good for channels with fading. Even with a single antenna, the channel offers some frequency diversity; however, the code cannot efficiently exploit it. What the CFC based precoding does is essentially to make the effective channel (as seen by the Reed-Muller code) look more like an AWGN channel so that the Reed-Muller code works better. Adding additional antennas has substantially the same effect:

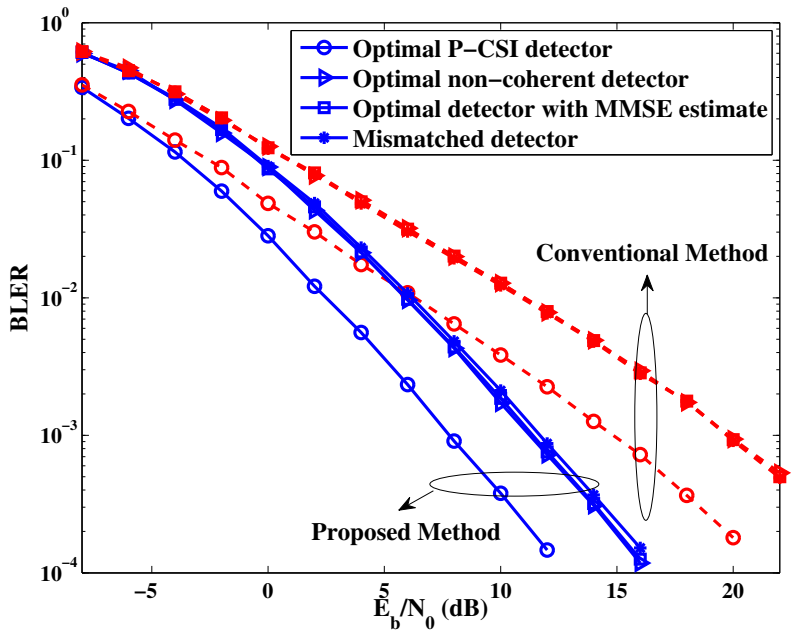

(b) MMSE estimate, $(20,13)$ - Reed-Muller code.

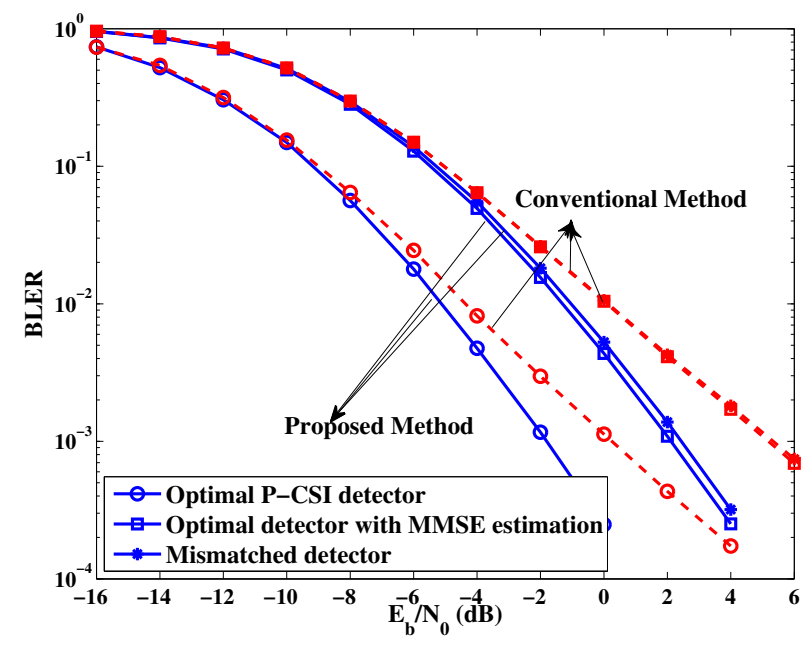

Figure 7. Performance comparison of the proposed method and the conventional coding method for the case when the receiver has two antennas. Here we used the $(20,13)$-Reed-Muller code with soft-decision decoding and MMSE estimation. $N_{0}$ value is assumed to be perfectly known at the receiver. We applied MRC for the signals received on two receive antenna branches.

the effective channel as seen by the Reed-Muller code becomes closer to an AWGN channel. Therefore, when the base station has more antennas, using the CFC based precoding offers diminishing returns.

\section{CONCLusions}

We have proposed improvements to the PUCCH format 2 control signaling in the uplink of an LTE system. The proposed method extracts the frequency diversity inherent in the channel by using the complex-field code to spread the information across the resources. This study reveals that there is a need for the design of short codes over $\mathrm{GF}(2)$ which can extract the diversity advantage available in wireless channels. For the users on cell-edge, for whom the channel estimation quality 
will be poor, it might be useful to use the optimal non-coherent detectors.

\section{REFERENCES}

[1] E. Dahlman, S. Parkvall, J. Sköld, and P. Beming, 3G Evolution- HSPA and LTE for Mobile Broadband, 2nd ed., Academic Press, 2008.

[2] D. Astély et al., "LTE: The evolution of mobile broadband," IEEE Comm. Magazine, vol. 47, pp. 44-51, April 2009.

[3] I. L. J. Da Silva et al., "A new multi-user receiver for PUCCH LTE format 1 ," Proc. of IEEE SPAWC, June 2010

[4] I. L. J. da Silva and A. L.F. de Almeida, "Multiuser receiver for PUCCH signaling with transmit diversity," Proc. of IEEE SPAWC, June 2011.

[5] Y. Wu, D. Danev, and E. G. Larsson, " Improved detection of ACK/NACK messages in the LTE uplink control channel," Proc. of IEEE VTC, May 2012.

[6] J. Puttonen, J. Kurjenniemi, O. Alanen, and T. Henttonen, "On improving the radio resource control signaling reliability in LTE uplink," Proc. of IEEE VTC, May 2011.

[7] M. R. Raghavendra, S. Nagaraj, K. V. Pradap, and P. Fleming, "Robust channel estimation and detection for uplink control channel in 3GPP LTE," Proc. of IEEE Globecom, Nov. 2009.

[8] T. V. K. Chaitanya, E. G. Larsson, and N. Wiberg, "Improved error protection for uplink control signaling in 3GPP-LTE via complex-field coding," Proc. of IEEE VTC, May 2010.

[9] E. G. Larsson, R. Thobaben, and G. Wang, " On diversity combining with unknown channel state information and unknown noise variance," Proc. of IEEE WCNC, Apr. 2010.

[10] 3GPP TS 36.212, Multiplexing and Channel Coding for Evolved UTRA, v.8.6.0., March 2009.

[11] 3GPP TS 36.211, Physical Channels and Modulation for Evolved UTRA, v.8.6.0., March 2009.

[12] J. Boutros and E. Viterbo, "Signal space diversity, a power- and bandwidth-efficient diversity technique for the Rayleigh fading channel," IEEE Trans. Inform. Theory, vol. 44, pp. 1453-1467, July 1998.

[13] Z. Li, Y. Xin, and G. B. Giannakis, "Linear constellation precoding for OFDM with maximum multipath diversity and coding gains," IEEE Trans. Comm., vol. 51, pp. 416-427, March 2003.

[14] G. Taricco and E. Bigleri, "Space-time decoding with imperfect channel estimation," IEEE Trans Wireless Commun., vol. 4, pp. 1874-1888, July 2005.

[15] E. G. Larsson and J. Jaldén, "Soft MIMO detection at fixed complexity via partial marginalization," IEEE Trans. Signal. Proc., vol. 56, pp. 33973407, Aug. 2008.

[16] Y. Be'ery and J. Snyders, "Optimal soft decision block decoders based on fast Hadamard transform," IEEE Trans. Inform. Theory, vol. 32, pp. 355-364, May 1986.

[17] T. Söderström, Discrete-time Stochastic Systems - Estimation and Control, second edition, Springer-Verlag, London, UK, 2002.

[18] 3GPP TS 36.101, User Equipment (UE) radio transmission and reception for Evolved -UTRA, v.8.6.0., June 2009.

[19] Recommendation ITU-R M.1225, Guidelines for evaluation of radio transmission technologies for IMT-2000, 1997.

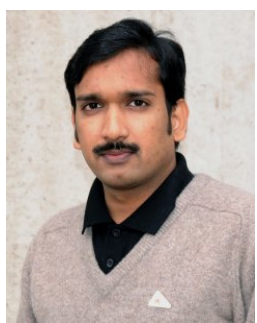

Tumula V. K. Chaitanya received the M.E. degree in telecommunications engineering from the Indian Institute of Science, Bangalore, India, in June 2005. $\mathrm{He}$ is currently working toward the Ph.D. degree with the Division of Communication Systems, Department of Electrical Engineering, Linköping University, Linköping, Sweden. From July 2005 to December 2008, he was with Beceem Communications Pvt. Ltd., Bangalore, as a Senior Design Engineer. His research interests include resource allocation in wireless networks, cooperative communications and signal processing algorithms for wireless communications.

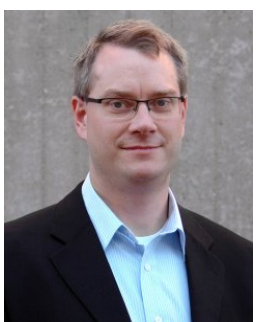

Erik G. Larsson (SM'10) received the Ph.D. degree from Uppsala University, Uppsala, Sweden, in 2002. He was an Associate Professor (Docent) with the Royal Institute of Technology, Stockholm, Sweden, and an Assistant Professor with both the University of Florida, Gainesville, and the George Washington University, Washington, DC. Since 2007, he has been a Professor and the Head of the Division for Communication Systems, Department of Electrical Engineering, Linköping University, Linköping, Sweden. He has published some 70 journal papers, is a coauthor of the textbook Space-Time Block Coding for Wireless Communications (Cambridge Univ. Press, 2003), and is the holder of ten patents on wireless technology. His research interests include wireless communications and signal processing.

Dr. Larsson is a member of the Technical Committee on Sensor Array and Multichannel and the Technical Committee on Signal Processing for Communications of the IEEE Signal Processing Society. He has been the Associate Editor for several IEEE JOURNALS. He is an Associate Editor for the IEEE TRANSACTIONS ON COMMUNICATIONS. He is active in conference organization, most recently as the Technical Chair of the 2012 Asilomar Conference on Signals, Systems, and Computers and a Technical Program Cochair of the 2012 International Symposium on Turbo Codes and Iterative Information Processing. 